\title{
Analyticity Properties of the Correlation Functions for the Anisotropic Heisenberg Model
}

\author{
Ch.-Ed. Pfister \\ Seminar für Theoretische Physik, ETH Zürich, Zürich, Switzerland
}

Received November 27, 1974

\begin{abstract}
It is shown for the Heisenberg model that the correlation functions are analytic in $h$ and $T$ if $\operatorname{Re}(h) \neq 0$ and $T$ is positive.

\section{Introduction}

The analyticity properties of the Ising model, when there is no phase transition, were established by Lee and Yang $[6,11]$ and by Lebowitz and Penrose [5]. The theorem of Lee-Yang about the zeros of the partition function of the system plays a prominent part in these papers. The generalization of this famous theorem to the case of the Heisenberg model was made by Asano [1] and Suzuki-Fisher [10]. With the help of this generalization we obtain analogous results as those obtained by Lebowitz and Penrose for the Ising model: the correlation functions are analytic in $h$ and $T$ if $\operatorname{Re}(h) \neq 0$ and $T$ is positive. The proof follows closely that of Lebowitz and Penrose. We use essentially the theorem of Lee-Yang and the technique introduced by Asano [1]. Our proof is only valid if the total magnetization commutes with the Hamiltonian, and does not extend to the general case considered by Suzuki and Fisher [10].
\end{abstract}

\section{Notation and Definition of the Model}

The model is defined on the lattice $\mathbb{Z}^{\nu}$. With each point of the lattice we associate a spin $-1 / 2$, which we describe by a Hilbert space $\mathscr{H}_{i}$ isomorphic to $\mathbb{C}^{2}$, and by the Pauli matrices $\sigma_{i}^{x}, \sigma_{i}^{y}, \sigma_{i}^{z}$. We consider first a system restricted to a finite subset $\Lambda$ of $\mathbb{Z}^{v}$. The corresponding Hilbert space is $\mathscr{H}_{\Lambda}=\bigotimes_{i \in \Lambda} \mathscr{H}_{i}$ and we choose the Hamiltonian as follows:

with

$$
H_{\Lambda}=-\sum_{\substack{i \neq j \\ i, j \in \Lambda}} H(i, j)+h \sum_{i \in \Lambda}\left(\sigma_{i}^{z}+1\right)
$$

$$
H(i, j)=K(i-j)\left(\sigma_{i}^{x} \sigma_{j}^{x}+\sigma_{i}^{y} \sigma_{j}^{y}\right)+J(i-j) \sigma_{i}^{z} \sigma_{j}^{z} .
$$

In this formula $H(i, j)$ describes an interaction between two spins. The interaction will be a ferromagnetic one:

$$
J(x)=J(-x) \geqq 0, \quad K(x)=g(x) J(x)
$$


with

$$
-1 \leqq g(x)=g(-x) \leqq+1 .
$$

The function $g$ allows us to introduce some anisotropy in the coupling between the spins. It is, however, very important for the rest of the paper that (4) holds:

$$
\left[\sum_{i \in \Lambda} \sigma_{i}^{z}, H\right]=0 \text {. }
$$

The constant $h$ may be interpreted as a magnetic field. We impose also two conditions on the decrease of the interactions $K$ and $J$ for large distances:

$$
\begin{aligned}
& \sup _{\substack{|s|>r>0 \\
s \in \mathbb{Z}^{v}}} r^{v} J(s)=u(r) \rightarrow 0, r \rightarrow \infty, \\
& \sum_{x \neq 0} J(x)<\infty, \quad \sum_{x \neq 0}|K(x)|<\infty .
\end{aligned}
$$

Such conditions ensure the existence of the thermodynamic limit of the correlation functions $[4,7]$. When we take the thermodynamic limit, this means that we choose a sequence of finite subsets of $\mathbb{Z}^{v},\left(\Lambda_{n}\right)_{n \in \mathbb{N}}$ such that $\Lambda_{n} \subset \Lambda_{n+1}$ for all $n$ and for every finite subset $\Delta$ of $\mathbb{Z}^{v}$ there is a number $N(\Delta)$ with the property that $\Delta$ is contained in $\Lambda_{p}$ for all $p>N(\Delta)$.

We denote the partition function by

$$
P(h, T, \Lambda)=\operatorname{Tr}_{\mathscr{H}_{\Lambda}} \exp \left(-\beta H_{\Lambda}\right), \quad \beta=(k T)^{-1},
$$

and the correlation functions by

$$
\left\langle\sigma_{x_{1}}^{i_{1}} \ldots \sigma_{x_{m}}^{i_{m}}\right\rangle(h, T, \Lambda)=\operatorname{Tr}_{\mathscr{H}_{\Lambda}}\left(\sigma_{x_{1}}^{i_{1}} \ldots \sigma_{x_{m}}^{i_{m}} \exp \left(-\beta H_{\Lambda}\right)\right) / P(h, T, \Lambda),
$$

where $x_{1}, \ldots, x_{m}$ are $m$ sites of $\Lambda$ and $i_{j}=x, y$ or $z, 1 \leqq j \leqq m$.

Remark. All finite subsets $\Lambda$ which we shall consider have the property: If $x$ and $y$ are two points of $\Lambda$, then there exists a set of points $\left\{x_{0}, \ldots, x_{n}\right\}$ with $x_{i} \in \Lambda$, $x_{0}=x, x_{n}=y$ and where the spins at $x_{i}$ and $x_{i+1}$ interact with one another.

\section{Results and Proofs}

If $\Lambda$ is a finite set, $H_{A}$ is a matrix and we may without difficulty consider complex values of $h$. Asano showed under the hypothesis $3 \mathrm{a}$ ) and $3 \mathrm{~b}$ ) that $P(h, T, \Lambda) \neq 0$ if $\operatorname{Re}(h) \neq 0$. On the other hand it is easy to see that $P(0, T, \Lambda) \neq 0$. We may thus define $\left\langle\sigma_{x_{1}}^{i_{1}} \ldots \sigma_{x_{m}}^{i_{m}}\right\rangle(h, T, \Lambda)$ if $\operatorname{Re}(h) \neq 0$ or $h$ real. Our first result is

Theorem I. For the model defined above [in particular if (3a), (3b), (5a), and (5b) hold].

1) If $T$ is a positive fixed number

$$
\lim _{\Lambda \rightarrow \infty}\left\langle\sigma_{x_{1}}^{i_{1}} \ldots \sigma_{x_{m}}^{i_{m}}\right\rangle(h, T, \Lambda)=\left\langle\sigma_{x_{1}}^{i_{1}} \ldots \sigma_{x_{m}}^{i_{m}}\right\rangle(h, T)
$$

converges locally uniformly in $h$, both when $\operatorname{Re}(h)>0$ and when $\operatorname{Re}(h)<0$.

2) The function $\left\langle\sigma_{x_{1}}^{i_{1}} \ldots \sigma_{x_{m}}^{i_{m}}\right\rangle(h, T)$ is analytic in $h$ and $T$ in the region $\operatorname{Re}(h)>0$ (and $\operatorname{Re}(h)<0$ ) and $T$ in a complex neighbourhood of the positive real axis.

Remark. If we introduce the variable $z=e^{-\beta h}$, then the domains of analyticity for the new variable become $|z|<1$ and $|z|>1$. 
Proof. We shall use the following result, which we shall prove later. in $\Lambda$

Lemma 1. Let $\Lambda$ be a finite subset of $\mathbb{Z}^{v}$. Then the inequality below is uniform

$$
\left|\left\langle\sigma_{x_{1}}^{i_{1}} \ldots \sigma_{x_{m}}^{i_{m}}\right\rangle(z, T, \Lambda)\right| \leqq\left(\frac{1+r}{1-r}\right)^{2 m} \text {, if }|z| \leqq r<1, r \quad \text { fixed }
$$

This lemma means that the family of analytic functions $\left\langle\sigma_{x_{1}}^{i_{1}} \ldots \sigma_{x_{m}}^{i_{m}}\right\rangle\left(z, T, \Lambda_{p}\right)$ with $p \in \mathbb{N}$ is a normal family [2] on the unit open $\operatorname{disc} E=\{z|| z \mid<1\}$. On the other hand it has been proved by Ginibre [4] that for every finite interval $I$ of the positive real axis there exists a complex neighbourhood $U$ of $z=0$ such that

$$
\lim _{p \rightarrow \infty}\left\langle\sigma_{x_{1}}^{i_{1}} \ldots \sigma_{x_{m}}^{i_{m}}\right\rangle\left(z, T, \Lambda_{p}\right)
$$

converges and defines an analytic function of $z$ and $T$ if $z \in U$ and $T \in I$. (The Hamiltonian in [4] is slightly different, but the proof of the above statement with our Hamiltonian is the same except for minor changes. The difference between the two Hamiltonians is a boundary term for the finite systems.) We apply then the theorem of Vitali [3] and we obtain the first result, the second one follows directly from the theorem of $[5$, p. 104]. Details of the proof may be found in the paper of Lebowitz and Penrose [5]. We obtain the same results in the case where $|z|>1$ using the symmetry of the model (see e.g. next section).

Let us suppose that there exists an arc $\gamma$ of the circumference of the unit circle $\{z|| z \mid=1\}$, on which, if $p$ is sufficiently large, $P\left(z, T, \Lambda_{p}\right)$ is non-zero. Without loss of generality we consider the case where $\gamma$ is given by the inequalities $-\varphi<\arg (z)$ $<+\varphi$ with $0<\varphi<\pi$. Under such assumptions the free energy is analytic in $z$ if $z \in \gamma$ in the thermodynamic limit. We extend this result to the correlation functions.

We consider the situation just described and we denote by $\delta \Gamma$ the circle which passes through the points $e^{i \varphi}$ and $e^{-i \varphi}$ and which is orthogonal to the unit circle. The open set which contains the point 1 and which has the boundary $\delta \Gamma$ is denoted by $\Gamma$. Then we obtain:

Theorem II. The first conclusion of Theorem I is true if we replace the unit disc $E$ by $\Gamma$. The function $\left\langle\sigma_{x_{1}}^{i_{1}} \ldots \sigma_{x_{m}}^{i_{m}}\right\rangle(z, T)$ coincides with that of Theorem I if $|z| \neq 1$.

Proof. If we use the notation of Theorem I we can prove

Lemma 2. The family $\left\langle\sigma_{x_{1}}^{i_{1}} \ldots \sigma_{x_{m}}^{i_{m}}\right\rangle\left(z, T, \Lambda_{p}\right), p \in \mathbb{N}$, is normal in $\Gamma$ when $p$ is large enough, i.e. when $P\left(z, T, \Lambda_{p}\right) \neq 0$ if $z \in \gamma$.

With this result the proof follows as before.

\section{Proof of the Lemmas 1 and 2}

\section{Introduction}

We discuss here the technique used to prove Lemmas 1 and 2. In these proofs the position of the zeros of some polynomials, and also their symmetry properties, take a prominent part. In this section we define these polynomials and give some of their properties. We prove the lemmas in the next section. 
We consider polynomials of $N$ complex variables. It is convenient to use the notation of Ruelle [8]. We introduce a finite set $\Omega$ of $N$ elements denoted by letters $x, y, \ldots$. We associate a complex variable $z_{x}$ to each $x$ of $\Omega$. If $X$ is a subset of $\Omega$, then $z_{X}$ is the set of variables $\left\{z_{x} \mid x \in X\right\}$ and $z^{X}=\prod_{x \in X} z_{x}$. We put $z^{y} \equiv 1$. Let $\mathscr{E}(\Omega)$ be the set of all complex-valued functions $f$ defined on the subsets of $\Omega$.

$$
f: X \subset \Omega \mapsto f(X) \in \mathbb{C} .
$$

Let $\mathscr{P}(\Omega)$ be the set of all complex polynomials with $N$ complex variables, linear in each $z_{x}$. Clearly there is a one-to-one correspondence between $\mathscr{E}(\Omega)$ and $\mathscr{P}(\Omega)$ :

$$
f \in \mathscr{E}(\Omega) \mapsto P_{f}\left(z_{\Omega}\right)=\sum_{X \subset \Omega} f(X) z^{X} \in \mathscr{P}(\Omega) .
$$

On the set $\mathscr{E}(\Omega)$ we define the transformation $D(x, y)$ when $x \neq y$ :

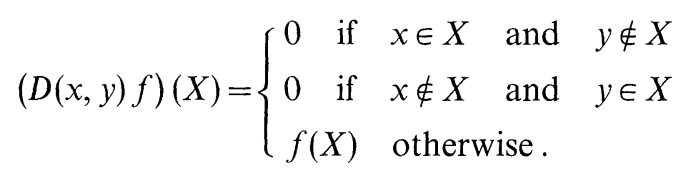

The corresponding transformation on $\mathscr{P}(\Omega)$ is

$$
\left(D\left(z_{x}, z_{y}\right) P_{f}\right)\left(z_{\Omega}\right)=P_{D(x, y) f}\left(z_{\Omega}\right) .
$$

This last operation is not exactly the contraction of Asano $[1,8,9]$. If we write explicitely only the variables $z_{x}$ and $z_{y}$, then we obtain

$$
P_{f}\left(z_{\Omega}\right)=a z_{x} z_{y}+b z_{x}+c z_{y}+d \vdash_{D\left(z_{x}, z_{y}\right)}^{\longrightarrow} a z_{x} z_{y}+d .
$$

The class $L(\Omega)$ of polynomials, which interest us, consists of all polynomials of $\mathscr{P}(\Omega)$, which satisfy the property $E$

$$
E: P_{f}\left(z_{\Omega}\right)=0 \text { and }\left|z_{x}\right| \leqq 1, \forall x \in \Omega \text { implies }\left|z_{x}\right|=1, \forall x \in \Omega .
$$

as also a symmetry property $S$ :

$$
S: f(X)=f(\Omega-X)^{*}, \quad \forall X \subset \Omega,
$$

where * represents complex conjugation.

Remarks. The property $E$ is equivalent to:

$$
\text { if } P\left(z_{\Omega}\right)=0 \text { and }\left|z_{y}\right| \leqq 1 \quad \forall y \in \Omega-\{x\} \text { and if } \exists y^{\prime} \in \Omega-\{x\}
$$

such that $\left|z_{y^{\prime}}\right|<1$, then $z_{x}$ is such that $\left|z_{x}\right|>1$.

The property $S$ means:

$$
\left(P_{f}\left(z_{\Omega}\right)\right)^{*}=\sum_{X \subset \Omega} f(\Omega-X)^{*} z^{* \Omega-X}=z^{* \Omega} P_{f}\left(\left(z^{*}\right)_{\Omega}^{-1}\right) .
$$

Proposition. The class $L(\Omega)$ is stable under the transformation $D\left(z_{x}, z_{y}\right)$.

Proof. The property $S$ is evidently conserved [see (10)]. The property $E$ is also conserved (Proposition 3.3, [8]). 
We note also two simple facts:

I) If $P_{f}\left(z_{\Omega}\right) \in \mathscr{P}(\Omega)$, we associate to $P_{f}$ a polynomial $Q_{f}$ in one complex variable $z$ by setting

$$
Q_{f}(z)=P_{f}(z, \ldots, z) \equiv a_{0} \prod_{i=1}^{N}\left(q_{i}-z\right), N=|\Omega| .
$$

We notice immediately that

$$
Q_{D(x, y) f}(z)=a_{0} \prod_{i=1}^{N}\left(\hat{q}_{i}-z\right) .
$$

II) If $P_{f}\left(z_{\Omega}\right) \in L(\Omega)$ and if we associate to each $x \in \Omega$ a complex number $\omega_{x}$ of unit modulus, then we can introduce new variables $\bar{z}_{x}=\omega_{x} z_{x}$ and define $P_{f}\left(\bar{z}_{\Omega}\right) \equiv P_{\bar{f}}\left(z_{\Omega}\right)$. Then

$$
Q_{\bar{f}}(z)=a_{0}\left(\prod_{x \in \Omega} \omega_{x}\right) \prod_{i=1}^{N}\left(\bar{q}_{i}-z\right) \equiv \bar{a}_{0} \prod_{i=1}^{N}\left(\bar{q}_{i}-z\right)
$$

and

$$
\bar{f}(X)=f(X) \prod_{y \in X} \omega_{y} .
$$

In particular $P_{\bar{f}}\left(z_{\Omega}\right)$ satisfies the property $E$ and the symmetry property $S_{\omega}$ :

$$
\bar{f}(X)=\omega \bar{f}(\Omega-X)^{*}, \quad \omega=\prod_{x \in \Omega} \omega_{x} .
$$

\section{Proof of the Lemmas 1 and 2}

Lemma 1. We consider the explicit case $i_{1}=x$ and $i_{2}=y$; the generalization to other correlation functions is immediate. The fact that $\Lambda$ is a subset of $\mathbb{Z}^{v}$ is unimportant. Therefore $\Lambda$ is here a set with $N$ elements $\{1, \ldots, N\}$ and we write 1 respectively 2 instead of $x_{1}$ respectively $x_{2}$ etc. We must show

$$
\sup _{|z| \leqq r<1}\left|\left\langle\sigma_{1}^{x} \sigma_{2}^{y}\right\rangle(z, T, \Lambda)\right| \leqq\left(\frac{1+r}{1-r}\right)^{4} .
$$

The proof consists of expressing $\left\langle\sigma_{1}^{x} \sigma_{2}^{y}\right\rangle(z, T, \Lambda)$ as a sum of four terms; each term is a quotient of polynomials, which possess the properties described in the last section. Then we use Remarks I and II in order to obtain the desired result.

\section{A. Definition of Four Polynomials}

In $\mathscr{H}_{A}$ we introduce the vectors $\left|\left\{s_{j}\right\}\right\rangle=\left|s_{1}\right\rangle \otimes \cdots \otimes\left|s_{N}\right\rangle$ defined by $\sigma_{j}^{z}\left|s_{j}\right\rangle$ $=s_{j}\left|s_{j}\right\rangle$ with $s_{j}= \pm 1$. These vectors form a basis in $\mathscr{H}_{\Lambda}$ and we index them by the subsets of $\Lambda$ :

$$
\left|\left\{s_{j}\right\}\right\rangle=|X\rangle: i \in X \Leftrightarrow s_{i}=+1 .
$$

The partition function becomes

$$
P(z, T, \Lambda)=\sum_{X \subset \Lambda}\left\langle X\left|\exp \left(-\beta H_{\Lambda}\right)\right| X\right\rangle, \beta=(k T)^{-1}, z=e^{-\beta h} .
$$


The operator $M=\sum_{i \in \Lambda}\left(\sigma_{i}^{z}+1\right)$ commutes with $H_{\Lambda} \equiv H_{0}+h M$. Therefore

$$
P(z, T, \Lambda)=\sum_{X \subset \Lambda}\left\langle X\left|\exp \left(\frac{-\beta h M}{2}\right) \exp \left(-\beta H_{0}\right) \exp \left(\frac{-\beta h M}{2}\right)\right| X\right\rangle
$$

is a polynomial in $z$. Trotter's formula allows us to write

$$
\exp \left(-\beta H_{0}\right)=\lim _{n \rightarrow \infty}\left(\prod_{i \neq j} \exp \left(\frac{\beta}{n} H(i, j)\right)\right)^{n} \equiv \lim _{n \rightarrow \infty} A_{n}
$$

with $H(i, j)$ given by (2). We make now the connection with the previous section: Let $\Lambda$ and $\Lambda^{\prime}$ be two copies of the set $\{1, \ldots, N\}$. We distinguish the elements or the subsets of $\Lambda^{\prime}$ by '. We define then $\Omega$ as the disjoint union of $\Lambda$ and $\Lambda^{\prime}$ and we write the subsets of $\Omega$ by the pairs $\left(X, Y^{\prime}\right)$ with $X \subset \Lambda$ and $Y^{\prime} \subset \Lambda^{\prime}$. Let $f_{n}$ be the function of $\mathscr{E}(\Omega)$ defined by

$$
f_{n}\left(X, Y^{\prime}\right)=\left\langle X\left|A_{n}\right| Y^{\prime}\right\rangle,
$$

and the corresponding polynomial

$$
P_{f_{n}}\left(z_{\Omega}\right)=P_{f_{n}}\left(z_{\Lambda}, z_{\Lambda^{\prime}}\right)=\sum_{\substack{X \subset A \\ Y^{\prime} \subset A^{\prime}}} z^{X} f_{n}\left(X, Y^{\prime}\right) z^{Y^{\prime}}
$$

we first define $g_{n} \in \mathscr{E}(\Omega)$ :

$$
g_{n}=D\left(3,3^{\prime}\right) \ldots D\left(N, N^{\prime}\right) f_{n}
$$

then we construct two polynomials in four variables $z_{1}, z_{1^{\prime}}, z_{2}, z_{2}$, and one complex parameter $w$ :

$$
P_{n}^{1}\left(z_{1}, z_{2}, z_{1^{\prime}}, z_{2^{\prime}} ; w\right)=D\left(z_{1}, z_{1^{\prime}}\right) D\left(z_{2}, z_{2^{\prime}}\right) P_{g_{n}}\left(z_{\Lambda}, z_{A^{\prime}}\right)
$$

and we put $z_{3}=z_{3^{\prime}}=\cdots=z_{N}=z_{N^{\prime}}=w$,

$$
P_{n}^{2}\left(z_{1}, z_{2}, z_{1^{\prime}}, z_{2^{\prime}} ; w\right)=P_{g_{n}}\left(z_{\Lambda}, z_{\Lambda^{\prime}}\right)
$$

and we put $z_{3}=z_{3^{\prime}}=\cdots=z_{N}=z_{N^{\prime}}=w$.

Finally we introduce

(cf. Remark I).

$$
\begin{aligned}
& Q_{n}^{1}(z ; w)=P_{n}^{1}(z, z, z, z ; w)=a_{0}(w) \prod_{i=1}^{4}\left(\hat{q}_{i}(w)-z\right), \\
& Q_{n}^{2}(z ; w)=P_{n}^{2}(z, z, z, z ; w)=a_{0}(w) \prod_{i=1}^{4}\left(q_{i}(w)-z\right),
\end{aligned}
$$

B. Relation between the Polynomials (27a), (27b), (28a), and (28b), and $\left\langle\sigma_{1}^{x} \sigma_{2}^{y}\right\rangle(z, T, \Lambda)$

We see immediately, comparing (22) and (28b), that

$$
P(z, T, \Lambda)=\lim _{n \rightarrow \infty} Q_{n}^{1}(z ; z),
$$

because

$$
\prod_{i=1}^{N} D\left(i, i^{\prime}\right) f_{n}\left(X, Y^{\prime}\right)=\left\{\begin{array}{lll}
0 & \text { if } & X \neq Y \\
f_{n}(X, X) & \text { if } & X=Y .
\end{array}\right.
$$


On the other hand we compute the expression

$$
\begin{gathered}
\operatorname{Tr}_{\mathscr{H}_{\Lambda}}\left[\sigma_{1}^{x} \sigma_{2}^{y} \exp \left(\frac{-\beta h M}{2}\right) \exp \left(-\beta H_{0}\right) \exp \left(\frac{-\beta h M}{2}\right)\right] \\
=\lim _{n \rightarrow \infty} \operatorname{Tr}_{\mathscr{H}_{\Lambda}}\left[\sigma_{1}^{x} \sigma_{2}^{y} \exp \left(\frac{-\beta h M}{2}\right) A_{n} \exp \left(\frac{-\beta h M}{2}\right)\right]
\end{gathered}
$$

with the aid of the basis

$$
\begin{aligned}
& \left.\mid\left\{s_{j}\right\}\right)=\left|s_{1}\right\rangle^{x} \otimes\left|s_{2}\right\rangle^{y} \otimes\left|s_{3}\right\rangle \otimes \cdots \otimes\left|s_{N}\right\rangle \\
= & \left.\mid s_{1}, s_{2}, X\right) \quad \text { with } \quad X \subset \tilde{\Lambda}=\Lambda-\{1,2\} .
\end{aligned}
$$

We have used the following vectors

$$
\begin{aligned}
& \left|s_{1}\right\rangle^{x}=\frac{1}{\sqrt{2}}\left(s_{1}|1\rangle+|-1\rangle\right), s_{1}= \pm 1 \\
& \left|s_{2}\right\rangle^{y}=\frac{1}{\sqrt{2}}\left(-i s_{2}|1\rangle+|-1\rangle\right), s_{2}= \pm 1
\end{aligned}
$$

which satisfy

$$
\sigma_{1}^{x}\left|s_{1}\right\rangle^{x}=s_{1}\left|s_{1}\right\rangle^{x}, \sigma_{2}^{y}\left|s_{2}\right\rangle^{y}=s_{2}\left|s_{2}\right\rangle^{y}
$$

Hence we obtain

$$
\begin{aligned}
& \operatorname{Tr}_{\mathscr{H}_{\Lambda}}\left[\sigma_{1}^{x} \sigma_{2}^{y} \exp \left(\frac{-\beta h M}{2}\right) A_{n} \exp \left(\frac{-\beta h M}{2}\right)\right] \\
& =\sum_{X \subset \tilde{\Lambda}}(1,1, X|\ldots| 1,1, X)-\sum_{X \subset \tilde{\Lambda}}(-1,1, X|\ldots|-1,1, X) \\
& -\sum_{X \subset \tilde{\Lambda}}(1,-1, X|\ldots| 1,-1, X)+\sum_{X \subset \tilde{\Lambda}}(-1,-1, X|\ldots|-1,-1, X)
\end{aligned}
$$

[... represents the expression between the square brackets on the left hand side of (35)].

Using (33) and the definition of $P_{n}^{2}$ we see that

$$
4 \sum_{X \subset \tilde{A}}(1,1, X|\ldots| 1,1, X)=P_{n}^{2}(z,-i z, z,-i z ; z)
$$

and the three other terms in (35) have analogous expressions.

$$
\text { C. Estimation of }\left\langle\sigma_{1}^{x} \sigma_{2}^{y}\right\rangle(z, T, \Lambda)
$$

We use now the result proved by Asano [1]:

$$
P_{f_{n}}\left(z_{\Omega}\right) \in L(\Omega) \text {. }
$$

We thus obtain using Remarks I and II

$$
P_{n}^{2}(z,-i z, z,-i z ; w)=\bar{a}_{0}(w) \prod_{i=1}^{4}\left(\bar{q}_{i}(w)-z\right) \text {. }
$$


Hence the $n^{\text {th }}$ approximation of $\left\langle\sigma_{1}^{x} \sigma_{2}^{y}\right\rangle(z, T, \Lambda)$ is the sum of four terms of the following type:

$$
\frac{1}{4} \frac{\bar{a}_{0}(z)}{a_{0}(z)} \prod_{i=1}^{4} \frac{\bar{q}_{i}(z)-z}{\hat{q}_{i}(z)-z} .
$$

If $|w| \leqq 1$, then $\left|\bar{q}_{i}(w)\right| \geqq 1,\left|\hat{q}_{i}(w)\right| \geqq 1$ and if $|w|=1$, then $\left|\bar{q}_{i}(w)\right|=\left|\hat{q}_{i}(w)\right|=1$.

Consequently

$$
\begin{gathered}
\sup _{|z| \leqq r<1} \frac{1}{4}\left|\frac{\bar{a}_{0}(z)}{a_{0}(z)}\right| \prod_{i=1}^{4}\left|\frac{\bar{q}_{i}(z)-z}{\hat{q}_{i}(z)-z}\right| \\
\leqq \sup _{\substack{|z| \leqq r<1 \\
|w|=1}} \frac{1}{4} \prod_{i=1}^{4}\left|\frac{\bar{q}_{i}(w)-z}{\hat{q}_{i}(w)-z}\right| \leqq \frac{1}{4}\left(\frac{1+r}{1-r}\right)^{4} .
\end{gathered}
$$

This last expression does not depend on $n$ and $\Lambda$. Hence the lemma is proved.

Lemma 2. We use the same notation as before. Let $P_{1}$ and $P_{2}$ be two interior points of the arc $\gamma$ and $k$ be the closed disc whose boundary is the circle passing through $P_{1}$ and $P_{2}$ and orthogonal to the unit circle. We introduce two subsets of $k: k_{1}=\{z \in k|| z \mid \geqq 1\}$ and $k_{2}=\{z \in k|| z \mid \leqq 1\}$. By the transformation $z \mapsto\left(z^{*}\right)^{-1}$ we have $k_{1} \mapsto k_{2}$. Let us take now any interior compact subset $k^{\prime}$ of $k$ and we put $d\left(k^{\prime}, \delta k\right) \equiv d>0$, where $d\left(k^{\prime}, \delta k\right)$ is the distance between $k^{\prime}$ and the boundary $\delta k$ of $k$. Let us consider $\left\langle\sigma_{1}^{x} \sigma_{2}^{y}\right\rangle\left(z, T, \Lambda_{p}\right)$. By assumption it is possible to find an integer $N(p, k)$ such that if $n>N(p, k) Q_{n}^{1}(z ; z) \neq 0$ for $z \in k \cap \gamma$. On the other hand $P_{n}^{1}\left(z_{\Lambda}, z_{\Lambda^{\prime}}\right) \neq 0$ if all $\left|z_{i}\right|<1$ or all $\left|z_{i}\right|>1$. We may apply the proposition p. 268 of [8] and hence we find

$$
P_{n}^{1}\left(z_{1}, z_{2}, z_{1^{\prime}}, z_{2^{\prime}} ; w\right) \neq 0 \text { if } z_{1}, z_{2}, z_{1^{\prime}}, z_{2^{\prime}}, \quad \text { and } w \in k
$$

In particular $Q_{n}^{1}(z ; w) \neq 0$ if $z$ and $w \in k$ therefore

$$
\min _{\substack{z \in k^{\prime} \\ w \in k}}\left|\hat{q}_{i}(w)-z\right| \geqq d\left(k^{\prime}, \delta k\right)=d .
$$

This estimation is independent of $p$ and $n$. If $w \in k_{1}$, we know that $\left|\bar{q}_{i}(w)\right| \leqq 1$; hence

$$
\max _{\substack{w \in k_{1} \\ z \in k^{\prime}}}\left|\bar{q}_{i}(w)-z\right| \leqq \phi\left(\overline{E \cup k_{1}}\right) \equiv \delta,
$$

where $\phi\left(\overline{E \cup k_{1}}\right)$ is the diameter of $\overline{E \cup k_{1}}$.

Finally we obtain

$$
\sup _{z \in k^{\prime} \cap k_{1}}\left|\left\langle\sigma_{1}^{x} \sigma_{2}^{y}\right\rangle\left(z, T, \Lambda_{p}\right)\right| \leqq 4\left(\frac{\delta}{d}\right)^{4} .
$$

We extend the validity of this last estimate using the symmetry properties. The symmetry $S$ of $P_{n}^{1}\left(z_{1}, z_{2}, z_{1^{\prime}}, z_{2^{\prime}} ; w\right)$ allows us to write

$$
\begin{gathered}
\left(a_{0}(w) \prod_{i=1}^{4}\left(\hat{q}_{i}(w)-z\right)\right)^{*} \\
=\left(w^{*}\right)^{2 N-4}\left(z^{*}\right)^{4} a_{0}\left(w^{*-1}\right) \prod_{i=1}^{4}\left(\hat{q}_{i}\left(w^{*-1}\right)-z^{*-1}\right)
\end{gathered}
$$


and the symmetry $S_{-1}$ of $P_{n}^{2}\left(z_{1},-i z_{2}, z_{1^{\prime}},-i z_{2^{\prime}} ; w\right)$

$$
\begin{gathered}
\left(\bar{a}_{0}(w) \prod_{i=1}^{4}\left(\bar{q}_{i}(w)-z\right)\right)^{*} \\
=-1\left(w^{*}\right)^{2 N-4}\left(z^{*}\right)^{4} \bar{a}_{0}\left(w^{*-1}\right) \prod_{i=1}^{4}\left(\bar{q}_{i}\left(w^{*-1}\right)-z^{*-1}\right) .
\end{gathered}
$$

Hence (43) is valid for all $z$ in $k^{\prime}$ and the lemma is proved.

Acknowledgements. We should like to thank Professor W. Hunziker for setting this problem his interest and his constant encouragements. We should like also to thank Professor G. Gallavotti and Dr. W. Wresziniski for very fruitful discussions and Dr. D. Webber for reading the manuscript.

\section{References}

1. Asano, T.: J. Phys. Soc. Japan 29, 350 (1970)

2. Ahlfors, L. V.: Complex analysis. New York: McGraw-Hill 1966

3. Behnke,H., Sommer, F.: Theorie der analytischen Funktionen einer komplexen Veränderlichen. Berlin-Heidelberg-New York: Springer 1965

4. Ginibre, J.: Commun. math. Phys. 10, 140 (1968)

5. Lebowitz, J. L., Penrose, P.: Commun. math. Phys. 11, 99 (1968)

6. Lee, T.D., Yang, C. N.: Phys. Rev. 87, 404 (1952)

7. Ruelle, D.: Statistical mechanics. New York: Benjamin 1969

8. Ruelle, D.: Commun. math. Phys. 31, 265 (1973)

9. Ruelle, D.: Phys. Rev. Letters 26, 303 (1971)

10. Suzuki, M., Fisher, M.E.: J. Math. Phys. 12, 235 (1971)

11. Yang, C. N., Lee, T. D.: Phys. Rev. 87, 410 (1952)

Communicated by G. Gallavotti

Ch.-Ed. Pfister

Theoretische Physik der ETH

Honggerberg

CH-8049 Zürich, Switzerland 
